\title{
Cortical Morphometric Abnormality and Its Association with Working Memory in Children with Attention-Deficit/Hyperactivity Disorder
}

\author{
Fei-Fei Si ${ }^{1}$, Lu Liu ${ }^{1 凶}$, Hai-Mei Li', Li Sun ${ }^{1}$, Qing-Jiu Cao ${ }^{1}$, Hanna Lu², Yu-Feng Wang ${ }^{1}$, and Qiu-Jin Qian ${ }^{1 凶}$ \\ ${ }^{1}$ Peking University Sixth Hospital, Peking University Institute of Mental Health, NHC Key Laboratory of Mental Health (Peking University), \\ National Clinical Research Center for Mental Disorders (Peking University Sixth Hospital), Beijing, China \\ ${ }^{2}$ Department of Psychiatry, The Chinese University of Hong Kong, Hong Kong, China
}

Objective Attention-deficit/hyperactivity disorder (ADHD) is a common neurodevelopmental disorder in children and adolescents. The present study investigated the cortical morphology features and their relationship with working memory (WM).

Methods In the present study, a total of 36 medication naïve children with ADHD (aged from 8 to 15 years) and 36 age- and gendermatched healthy control (HC) children were included. The digit span test was used to evaluate WM. The magnetic resonance imaging (MRI) was used to examine the characteristics of cortical morphology. Firstly, we compared the cortical morphology features between two groups to identify the potential structural alterations of cortical volume, surface, thickness, and curvature in children with ADHD. Then, the correlation between the brain structural abnormalities and WM was further explored in children with ADHD.

Results Compared with the HC children, the children with ADHD showed reduced cortical volumes in the left lateral superior temporal gyrus (STG) $\left(\mathrm{p}=6.67 \times 10^{-6}\right)$ and left anterior cingulate cortex (ACC) $\left(\mathrm{p}=3.88 \times 10^{-4}\right)$. In addition, the cortical volume of left lateral STG was positively correlated with WM $(\mathrm{r}=0.36, \mathrm{p}=0.029)$.

Conclusion Though preliminary, these findings suggest that the reduced cortical volumes of left lateral STG may contribute to the pathogenesis of ADHD and correlate with WM in children with ADHD.

Psychiatry Investig 2021;18(7):679-687

Key Words ADHD, MRI, Cortical morphology, Working memory.

\section{INTRODUCTION}

Attention-deficit/hyperactivity disorder (ADHD) is one of the most common neurodevelopmental disorders in children and adolescents. ADHD occurs in $7.2 \%$ of children, ${ }^{1}$ characterized by impaired levels of inattention, hyperactivity, and

Received: September 22, 2020 Revised: November 21, 2020

Accepted: May 2, 2021

$\triangle$ Correspondence: Lu Liu, MD, PhD

Peking University Sixth Hospital, Peking Institute of Mental Health, NHC Key Laboratory of Mental Health (Peking University), National Clinical Research Center for Mental Disorders (Peking University Sixth Hospital), No.51, Hua Yuan Bei Lu, Haidian District, Beijing 100191, China

Tel: +86-10-62070258, E-mail: liulupku@bjmu.edu.cn

$\triangle$ Correspondence: Qiu-Jin Qian, MD, PhD

Peking University Sixth Hospital, Peking Institute of Mental Health, NHC Key Laboratory of Mental Health (Peking University), National Clinical Research Center for Mental Disorders (Peking University Sixth Hospital), No.51, Hua Yuan Bei Lu, Haidian District, Beijing 100191, China Tel: +86-10-62723701, E-mail: qianqiujin@bjmu.edu.cn

(c) This is an Open Access article distributed under the terms of the Creative Commons Attribution Non-Commercial License (https://creativecommons.org/licenses/bync/4.0) which permits unrestricted non-commercial use, distribution, and reproduction in any medium, provided the original work is properly cited. impulsivity which can affect their academic and social development. $^{2}$ The exact aetiology and pathophysiology of ADHD remains unclear. There is increasing evidence showing that the abnormalities in brain structure may play an important role in the pathophysiology of this disorder.,

Structural magnetic resonance imaging (sMRI) has been widely used to explore the differences in brain structure between childhood ADHD patients and healthy controls. Smaller total brain volume ${ }^{5,6}$ and structural abnormalities in frontostriatal, fronto-temporo-parietal and fronto-cerebellar networks have been found in children with $\mathrm{ADHD}{ }^{7}$ As far as brain cortical morphology's concerned, ADHD has been categorized as a delay in cortical maturation. ${ }^{8,9}$ For example, significantly smaller prefrontal cortical surface areas were found in children with ADHD. ${ }^{8,10}$ Along with a decrease in surface area of over $7 \%$ in both hemispheres, the children with ADHD also displayed a decrease in cortical folding bilaterally. ${ }^{11}$ Another sMRI study has contended that children with ADHD had global thinning of the cortex, particularly in the prefrontal and 
precentral regions. ${ }^{12}$ ADHD children had reduced cortical thickness in the left superior frontal, left orbito-frontal and left dorsal anterior cingulate cortex (ACC) and decreased gray matter volume in the left orbito-frontal, left middle frontal/ dorsolateral prefrontal, left middle temporal and left cerebellum in comparison to the control group. ${ }^{13}$

Brain structural abnormalities might lead to impairment of cognitive functions, including working memory (WM), which has been widely studied and implicated in the neuropsychological model of ADHD. ${ }^{14}$ In the existing literature, the relationship between brain structural abnormalities and WM were revealed for multiple psychiatric disorders. For instance, cortical thickness and surface area in the frontal, temporal, cingular and occipital brain regions were found to be significantly associated with cognitive function among first-episode psychosis patients, including WM. ${ }^{15}$ For bipolar disorder, negative relationship between right temporal pole thickness and WM was discovered. ${ }^{16}$ Moreover, a recent study indicated that WM was associated with larger volume and surface in frontotemporal regions across the psychosis spectrum. ${ }^{17}$ Higher gray matter volume in several regions of frontal lobe, cerebellum and temporal lobe was associated with better WM in adult ADHD ${ }^{18}$ As for children with ADHD, they often experience WM difficulties that may be associated with a worse outcome. It is those very common behaviors associated with WM deficits that might be the main complaints that lead children with ADHD to seek treatment in hospitals. ${ }^{19}$ Whilst a number of studies have examined brain structural abnormalities in children with ADHD, far fewer studies have further explored the association between the altered brain structures and deficit WM. The only one study that recruited 17 children with ADHD for cortical thickness analyses denoted a direct relationship between thinner cortical thickness of the left medial temporal cortex and deficient WM. ${ }^{20}$ In addition to cortical thickness, more dimensional brain cortical morphology indicators are worth exploring simultaneously to uncover the alteration in brain morphology associated with $\mathrm{ADHD}$, such as cortical volume, surface area, and cortical curvature. Cortical volume is the product of two independent components, cortical thickness and surface area. Decreased cortical volume might be associated with either a cortical thinning of the cortex or a decrease in the cortical surface area (or a combination of both). ${ }^{11}$ Cortical thickness is measured as the distance from the cortical white-gray matter boundary to the cortical surface (graycerebrospinal fluid boundary). ${ }^{21,22}$ The surface defining the boundary between gray matter and cerebrospinal fluid, or pial surface, was determined by deforming the gray/white surface outward. ${ }^{23,24}$ Cortical curvature assesses differences in small areas (peak of gyri and sulci). ${ }^{22}$ Studies on cortical volume showed that brain regions associated with cognition func- tion were more likely to show changes in ADHD. ${ }^{25}$ Reduced cortical surface area and thickness indicated that there is a delay in the maturation of brain in ADHD. ${ }^{8,26}$ Cortical curvature may be an incidental finding of uncertain clinical significance. ${ }^{22}$ In all, the analyses of these four different structural indicators simultaneously would help us to understand the characteristics of brain structural abnormalities in ADHD more comprehensively. ${ }^{11}$

The present study builds upon the limited research on cortical morphology in children with ADHD by involving 36 children with ADHD and 36 age- and gender- matched healthy controls to investigate the cortical morphology features (cortical volume, surface area, and cortical curvature) and their relationship with WM. We only included medication-naïve patients in this study to eliminate potential confounding effects of medication. ${ }^{5}$ We hypothesized that: 1) children with ADHD would show regional cortical morphology abnormalities, and 2) cortical morphology abnormalities would be associated with WM within the ADHD group.

\section{METHODS}

\section{Participants}

All ADHD cases were recruited from child psychiatric clinics of our hospital. Diagnoses of ADHD were conducted according to the DSM-IV criteria based on a semi-structured interview by experienced child psychiatrists using the Kiddie Schedule for Affective Disorders and Schizophrenia-Present and Lifetime Version (K-SADS-PL). ${ }^{27}$ The inclusion criteria were as follows: 1) DSM-IV ADHD diagnosis, 2) age between 8 and 15 years, 3 ) full-scale intelligence quotient (FSIQ) $\geq 80$ as measured by the Wechsler Intelligence Scale for Chinese Children-Revised (WISCC-R), ${ }^{28}$ 4) medication-naïve, 5) righthandedness, as assessed by the Chinese Handedness Inventory. ${ }^{29}$ The exclusion criteria for ADHD group were comorbidity with major neurological disorders, a diagnosis of schizophrenia, pervasive developmental disorder, tic disorder, emotional disorders, affective disorders, epilepsy, mental retardation, or other brain disorders, except for Learning Disorder (LD) and Disruptive Behavior Disorder (DBD). Forty-one ADHD children participated in the study. Five cases were excluded from analysis because of poor scan quality. Then, a total of 36 (28 males and 8 females) ADHD were enrolled for final analyses, including 19 ADHD inattentive type (ADHD-I), 1 ADHD hyperactive-impulsive type (ADHD-HI) and 16 ADHD combined type (ADHD-C) subjects. The mean age was 10.5 years $(\mathrm{SD}=1.8)$.

Healthy control (HC) subjects were all students from local schools, subjected to the Kiddie Schedule for Affective Disorders and Schizophrenia-Present and Lifetime Version (K- 
SADS-PL) by the same group of child psychiatrists that evaluated the ADHD group. None of the normal control subjects met the criteria for ADHD or any other psychiatric disorders. Finally, 36 age- and gender- matched individuals (28 males and 8 females) comprised the HC group. All normal control group were right-handed. The mean age was 10.5 years $(\mathrm{SD}=1.6)$.

This study and its procedures were approved by the Ethics Committee of Peking University Institute of Mental Health. The institutional review board (IRB) number is PKUH6 (2007)(39). After procedures had been explained, written informed consent was obtained from all subjects and their parents. All procedures have been performed in accordance with the ethical standards laid down in the 1964 Declaration of Helsinki and its later amendments.

\section{Psychiatric and neurocognitive assessments}

ADHD symptoms were evaluated using ADHD Rating Scale-IV (ADHD-RS-IV), which consists of 18 items according to DSM-IV for ADHD. ${ }^{30}$ Each symptom was scored on how often it occurred (i.e., if "never" presented a symptom, it would be coded as 0 ; if "occasionally," 1;"often," 2; and "always," 3. We employed the Digit Span Tests from the WISCCR to measure WM, involving two subtests: Digit Span Forward (DSF) and Digit Span Backward (DSB). DSF was used to assess simple verbal memory and attention span. ${ }^{31}$ DSB was applied for auditory working memory manipulation, testing the maximum number series an individual can aurally attend to, store and then repeat verbally in reverse order. ${ }^{31}$ DSF and DSB should be treated as different constructs. DSF emerged as a signifificant predictor for one measure of attention, ${ }^{32}$ while DSB was considered to be a measure of WM because it demanded both storage and manipulation in order to retain and repeat the number in reverse order. ${ }^{20,32}$ Because the digit span (DS) subtest of the Wechsler Intelligence Scale consists of DSF and DSB components that produce separate raw scores but are combined to produce a single scaled score. ${ }^{32}$ Raw scores of DSB can be used to compare group differences in WM. ${ }^{33}$ Therefore, the raw score of DSB and scaled score of DS were utilized in our study. ${ }^{20,34}$

\section{Structural MRI data acquisition and Image processing}

All imaging data were acquired on a Siemens Trio 3T scanner (Siemens, Erlangen, Germany) at the State Key Laboratory of Cognitive Neuroscience and Learning, Beijing Normal University. High-resolution 3D T1-weighted images were obtained using a magnetization-prepared rapid gradient echo (MP-RAGE) sequence: repetition time $(\mathrm{TR})=2,530 \mathrm{~ms}$; echo time $(\mathrm{TE})=3.39 \mathrm{~ms}$; inversion time $=1,100 \mathrm{~ms}$; flip angle $=7^{\circ}$; field of view $(\mathrm{FOV})=256 \times 256 \mathrm{~mm}$; matrix $=256 \times 256 ; 128$ slices, thickness=1.33 mm. MRI data were analyzed with atlas-based FreeSurfer software (http://surfer.nmr.mgh.harvard.edu, version 5.1.0). Freesurfer adopted a fully automated method to perform pre-processing steps including Talairach alignment, intensity normalization, and removal of skull and non-brain tissue with a hybrid watershed/surface deformation procedure, separation of the cerebellum and brainstem from the cerebrum and splitting of the left and right hemispheres. Each hemisphere was divided into 74 different cortical regions in compliance with the Destrieux's atlas. ${ }^{35}$ Images with motion-related artifacts were excluded. As for the quality control of the images, we followed the ENIGMA pipeline (http://enigma.ini.usc.edu/). All brain cortical regions with a volume, surface, thickness, or curvature $>1.5$ or $<1.5$ times the interquartile range were identified and visually inspected by overlaying their segmentations on the subjects' anatomical images. Only subjects with images for which segmentation were judged to be accurate were included to further analysis. Cortical volume, cortical thickness, cortical surface area, and curvature were measured applying Freesurfer on all the subjects.

\section{Statistical analysis}

Statistical analyses were conducted in SPSS, version 19 (IBM Corp., Armonk, NY, USA) and R (https://www.r-project.org/). Demographics and clinical characteristics were compared using two-tailed t-tests for continuous variables or chi-square $\left(\chi^{2}\right)$ tests in SPSS for categorical variables between patients and controls. Cortical volume, surface, thickness, and curvature were examined by the modified generalized linear model (GLM) in R, with age, sex, and FSIQ as covariates. Modified GLM, also known as glm 2 in $\mathrm{R}$, is a modified version of GLM in the stats package. It is identical to GLM except for minor modifications to change the default fitting method and more stable compared with GLM. ${ }^{36}$ Packages of "haven" and "glm2" in the RStudio (https://www.rstudio.com/products/rstudio/download/) were used in our present study to analyze the data in the sav file. To adjust the person-to-person variations in head size, total intracranial volume (ICV) (summed volumes of total grey matter, total white matter and cerebrospinal fluid compartment derived from Freesurfer's segmentation procedure) was set as a covariate. The level of significance was defined as $\mathrm{p}<0.05$. Considering the number of analyzed cortical regions $(n=74)$, Bonferroni corrections were performed with setting the adjusted significance at $\mathrm{p}<6.8 \times 10^{-4}(0.05 / 74)$.

We further explored the correlation between abnormal brain structures in children with ADHD and their WM using Pearson partial correlation with age, sex and ICV as covariates. A statistical threshold of $\mathrm{p}=0.05$ was employed for all correlational analyses. Taking into account of the number of analyzed cortical regions $(n=2)$ and the number of WM indi- 
cators $(\mathrm{n}=2)$, the adjusted significance was set to $\mathrm{p}<0.0125$ $(0.05 / 4)$.

\section{RESULTS}

\section{Demographic and clinical variables}

The demographic and clinical characteristics of children with ADHD and healthy controls were shown in Table 1.

The age $[(10.5 \pm 1.8)$ vs. $(10.5 \pm 1.6), \mathrm{p}=0.891]$ and gender $\left(\chi^{2}<0.01, \mathrm{p}=1.000\right)$ of participants in both children with ADHD and healthy controls were found no significant difference. HC subjects had a significantly higher FSIQ score than children with ADHD [(117.5 \pm 15.1$)$ vs. $(105.4 \pm 14.6), \mathrm{p}=0.001]$. Compared with HC subjects, significant group differences were observed on DSB and DS with age and sex as covariates. Children with ADHD performed significantly worse on DSB [(4.64 \pm 1.31$)$ vs. $(5.57 \pm 1.58), \mathrm{p}=0.001]$ and DS [(10.92 \pm 2.66$)$ vs. $\left.(13.20 \pm 3.08), \mathrm{p}=3.62 \times 10^{-4}\right]$ than controls.

\section{Cortical morphologic abnormality}

Children with ADHD had a significant reduction of cortical volume in the left lateral aspect of the superior temporal gyrus (STG) $\left(\mathrm{p}=6.67 \times 10^{-6}\right)$ and left ACC $\left(\mathrm{p}=3.88 \times 10^{-4}\right)$ compared with controls with age, sex, FSIQ and ICV adjusted. In addition, children with $\mathrm{ADHD}$ exhibited a nominal reduction of cortical surface in the right ACC, middle-anterior part of ACC and sulcus (aMCC), supramarginal gyrus, planum polare of the STG, temporal pole, medial orbital sulcus (olfactory sulcus), superior temporal sulcus, and the left aMCC, inferior segment of the circular sulcus of the insula, parietooccipital sulcus. However, these regions did not survive Bonferroni corrections (Table 2).

There were no significant between-group differences in cortical surface, thickness or curvature after correction for mul- tiple comparisons. Nominal reduction of cortical surface was found for children with ADHD in the bilateral cingulate cortices and the left parietal and temporal cortices before correction for multiple (Supplementary Table 1 in the online-only Data Supplement). In addition, children with ADHD manifested a nominal reduction of cortical thickness in the bilateral frontal and temporal cortices before correction for multiple (Supplementary Table 2 in the online-only Data Supplement). Children with ADHD showed a nominal decreased of cortical curvature in the right right orbital part of the inferior frontal gyrus before multiple corrections (Supplementary Table 3 in the online-only Data Supplement).

\section{Correlations between abnormal cortical volumes and WM}

The volume of left lateral aspect of the STG volume was positively correlated with DSB scores after controlling for age, sex and ICV ( $r=0.36, p=0.029)$. To be specific, the bigger the volume in children with ADHD was, the higher DSB scores were and the better WM was (Table 3). However, their correlation could not survive Bonferroni corrections. There were no correlations between the volume of ACC and DSB/DS scores.

\section{DISCUSSION}

As expected, the results of our present study provided strong evidence that children with ADHD showed cortical morphology abnormalities relative to healthy controls. Children with ADHD had smaller cortical volumes in the left lateral STG and left ACC. The decreased volume of the left lateral STG might be correlated with impaired WM in children with ADHD.

\section{Cortical volume in the left lateral STG}

The enrollment of temporal gyrus in ADHD has been illus-

Table 1. Demographic and clinical variables of ADHD and healthy controls

\begin{tabular}{|c|c|c|c|c|}
\hline & $\operatorname{ADHD}(\mathrm{N}=36)$ & Controls $(\mathrm{N}=36)$ & $t / \chi^{2}$ & $\mathrm{p}$ \\
\hline Sex (male/female) & $28 / 8$ & $28 / 8$ & $<0.01$ & $>0.999$ \\
\hline Age in year (mean $\pm S D)$ & $10.5 \pm 1.8$ & $10.5 \pm 1.6$ & 0.14 & 0.891 \\
\hline FSIQ (mean \pm SD) & $105.4 \pm 14.6$ & $117.5 \pm 15.1$ & -3.46 & 0.001 \\
\hline \multicolumn{5}{|l|}{ ADHD subtype (N, \%) } \\
\hline Inattentive type & $19(52.8)$ & NA & & \\
\hline Combined & $16(44.4)$ & NA & & \\
\hline Hyperactive-impulsive type & $1(2.8)$ & NA & & \\
\hline \multicolumn{5}{|l|}{ Digit span test (mean $\pm \mathrm{SD})$} \\
\hline DSB score & $4.64 \pm 1.31$ & $5.57 \pm 1.58$ & 11.64 & 0.001 \\
\hline DS score & $10.92 \pm 2.66$ & $13.20 \pm 3.08$ & 14.12 & $3.62 \times 10^{-4}$ \\
\hline
\end{tabular}

ADHD: attention-deficit/hyperactivity disorder, SD: standard devision, FSIQ: full-scale intelligence quotient, NA: not applicable, DSB: digit span backward, DS: digit span 
Table 2. Estimated regression parameters, standard errors, $t$ values, and $p$-values for the group differences in cortical volume

\begin{tabular}{|c|c|c|c|c|}
\hline & Estimate & Std.error & t value & p-value ${ }^{\mathrm{a}}$ \\
\hline \multicolumn{5}{|l|}{ Right hemisphere } \\
\hline Anterior cingulate cortex & 2.943 & 9.248 & 3.183 & 0.002 \\
\hline Middle-anterior part of anterior cingulate cortex and sulcus & 2.628 & 1.256 & 2.092 & 0.041 \\
\hline Supramarginal gyrus & 1.220 & 4.643 & 2.628 & 0.011 \\
\hline Planum polare of the superior temporal tyrus & 4.432 & 1.506 & 2.943 & 0.005 \\
\hline Temporal pole & 1.913 & 7.960 & 2.404 & 0.019 \\
\hline Medial orbital sulcus & 6.990 & 2.378 & 2.939 & 0.005 \\
\hline Superior temporal sulcus & 1.082 & 5.070 & 2.133 & 0.037 \\
\hline \multicolumn{5}{|l|}{ Left hemisphere } \\
\hline Anterior cingulate cortex & 3.140 & 8.307 & 3.780 & $3.88 \times 10^{-4 *}$ \\
\hline Middle-anterior part of anterior cingulate cortex and sulcus & 2.182 & 1.041 & 2.096 & 0.041 \\
\hline Lateral aspect of the superior temporal gyrus & 3.420 & 6.870 & 4.979 & $6.67 \times 10^{-6 *}$ \\
\hline Inferior segment of the circular sulcus of the insula & 5.092 & 1.737 & 2.932 & 0.005 \\
\hline Parieto-occipital sulcus & 2.410 & 1.042 & 2.313 & 0.025 \\
\hline
\end{tabular}

aage, sex, full-scale intelligence quotient (FSIQ) and total intracranial volume (ICV) as covariates; All p-values implied uncorrected p-values. *p-values survived Bonferroni corrections

Table 3. Correlations between the variables of the volume and DSB, DS

\begin{tabular}{|c|c|c|}
\hline & $\begin{array}{c}\operatorname{ADHD}(\mathrm{N}=36) \\
(\mathrm{r}, \mathrm{p})\end{array}$ & $\begin{array}{c}\text { Controls }(\mathrm{N}=35) \\
(\mathrm{r}, \mathrm{p})\end{array}$ \\
\hline \multicolumn{3}{|c|}{ Left lateral aspect of the superior temporal gyrus } \\
\hline DSB score & $0.36,0.029$ & $-0.11,0.519$ \\
\hline DS score & $0.27,0.118$ & $-0.22,0.206$ \\
\hline \multicolumn{3}{|c|}{ Left anterior cingulate cortex } \\
\hline DSB score & $0.295,0.080$ & $-0.226,0.191$ \\
\hline DS score & $0.068,0.693$ & $-0.308,0.072$ \\
\hline
\end{tabular}

age, sex and total intracranial volume (ICV) as covariates. DSB: digit span backward, DS: digit span, ADHD: attention-deficit/hyperactivity disorder

trated in previous studies. One recent study recruiting 18 children with ADHD showed decreased volume in the left middle temporal gyrus in children with ADHD. ${ }^{13}$ Boys with ADHD showed significantly lower gray volume in the right temporal regions. ${ }^{37}$ Some other studies also argued that children and adolescents with ADHD had reduced volume in the middle temporal gyrus, ${ }^{38}$ the bilateral temporal polar and occipital cortices and right superior temporal sulcus, ${ }^{25}$ or the right middle and inferior temporal gyri. ${ }^{39}$ Young adults with ADHD showed reduced volume in the right superior and middle temporal gyrus ${ }^{4}$ and higher fractional anisotropy in the right STG. ${ }^{40}$ Taken together with these preceding evidence, our present finding of the reduced volume in the left STG supported the importance of structural alteration of temporal gyrus in the etiology of ADHD. However, there was inconsistency on the exact location, which might be due to limited sample size or a gender-by-diagnosis interaction effect in the volume of brain abnormalities. ${ }^{41}$

It's worth noting that our findings of abnormalities in left STG in children with ADHD parallel with some AMRI findings. Children with ADHD displayed increased brain connectivity in the left STG after four weeks of equine-assisted activity and training that improved clinical symptoms of ADHD. ${ }^{42}$ Our study group has found that decreases in negative functional connectivity with the putamen-ROIs was seen in STG in medication-naïve children with ADHD relative to controls. ${ }^{43} \mathrm{An}$ event-related fMRI revealed significantly reduced brain activation in left superior temporal lobes in patients compared with healthy subjects, and that the activation differences in superior temporal lobes correlated inversely with response variability in control subjects but not in patients. ${ }^{44}$ Another fMRI study claimed that increased fractional amplitude of low-frequency fluctuations (fALFF) in the left STG was related to reductions in inattentive symptoms for children with ADHD treated with methylphenidate. ${ }^{45}$ Besides, adolescents with ADHD also presented increased activation in the STG during successful response inhibition. ${ }^{46}$ Interestingly, one study on quantifying patterns of brain activity between ADHD and their unaffected siblings also announced that STG is associated with risk for ADHD ${ }^{47}$ In summary, fMRI studies have expressed that abnormal functional relationships between STG and other brain regions may underlie the pathological basis of ADHD. ${ }^{43,48,49}$ The results showed that the left lateral STG which we identified structural deficits in the children ADHD group also manifested functional deficits. Further studies are needed to explore the neural alterations in children with $\mathrm{ADHD}$ 
by combining and fusing both brain structural and functional imaging data to achieve more comprehensive interpretation.

\section{Cortical volume in the left ACC}

The reduced volume in the left ACC offered strong evidence that the left ACC is abnormal in children with ADHD. One study provided consistent evidence of the structural deficits observed in the left ACC for untreated children with ADHD. ${ }^{5}$ A recent sMRI study including 18 children with ADHD and 18 healthy children also has identified that patients showed smaller volumes in the left ACC and the reductions of volume were correlated with patients' inattention. ${ }^{50} \mathrm{~A}$ meta-analysis of MRI studies, collecting 11 VBM studies from 2001 to 2011 in all, has found that untreated children with ADHD tended to show additional changes in the left ACC compared with treated children and healthy children. ${ }^{51}$ Interestingly, the meta-analysis also contended that adults with persistent ADHD symptoms were still characterized by volume reductions in the left and right ACC. Meanwhile, a study by Matsuo et al..$^{52}$ that adult ADHD patients presented volume in the left ACC. Several studies stated that structural abnormalities in ADHD occur before the age of 10 and can persist into adulthood. ${ }^{51,53,54}$ Therefore, these results resonate well with our finding reported here, which suggests that ADHD children show reduced volume in the left ACC. Inconsistent with our results, one previous study has argued that only the right ACC was significantly smaller in the treatment-naive children with ADHD-C compared with the treated children or healthy children..$^{55}$ However, further analyses from the same researchers have identified that the ADHD-C group had bilaterally smaller volumes of the ACC compared with ADHD-I or healthy children. ${ }^{56}$ This discrepancy might be attributable to ADHD subtypes ${ }^{56}$ or the use of medication ${ }^{5}$ effects in the volume of brain abnormalities. In order to exclude the possible influence of ADHD subtypes on the results, we initially analyzed the difference in cortical morphology among ADHD-I subtypes, ADHD-C subtypes and healthy controls. The preliminary analyses among three groups indicated differences in cortical volume in the left lateral aspect of the STG , with age, sex, FSIQ and ICV adjusted, which is consistent with the results between children with $\mathrm{ADHD}$ and $\mathrm{HC}$ subjects. Post-hoc analysis indicated that both ADHD-I and ADHD-C were with smaller cortical volume in the left lateral STG than HC, however, no significant difference was found between these two subtypes (the significant region is shown in the Supplementary Table 4 in the online-only Data Supplement, and visualized in the Supplementary Figure 1 in the online-only Data Supplement).

Furthermore, several functional MRI studies have indicated that children with ADHD demonstrated marked reduced activation in the ACC extending to the supplementary motor area during response inhibition ${ }^{57}$ or during attentional alerting. ${ }^{58}$ Likewise, a systematic review of fMRI and electrophysiological (electroencephalography, EEG) studies has declared that children with ADHD showed an enhanced activation in the ACC. ${ }^{59} \mathrm{~A}$ recent event-related potential source imaging study revealed reduced activation in the ACC children with ADHD during the stop-signal task. ${ }^{60}$ These findings largely echo the results in the current study. In a nutshell, our findings confirm the abnormality of the left ACC in children with ADHD.

\section{Cortical thickness, surface area and curvature}

In this study, there were no differences in cortical surface, thickness, and curvature between children with ADHD and healthy controls. For the cortical surface, smaller cortical surface areas bilaterally ${ }^{11}$ and prefrontal cortical surface areas ${ }^{8,10}$ have been indicated in children with ADHD; however, negative results have also been reported as in our present study. ${ }^{22}$ The inconsistency also existed for the studies of cortical thickness. some found that children with ADHD had decreased cortical thickness in the prefrontal, precentral and temporal regions; ${ }^{12,22,61}$ wheras, some not. ${ }^{11}$ As for cortical curvature, there were very few group differences. ${ }^{22}$ Another study also spotted no differences in cortical gyrification index (a measure comparable to curvature) between children with ADHD and healthy controls. ${ }^{8}$ Multiple factors might influence the consistency and reproducibility among different studies. Firstly, the small sample size might influence the statistical power and lead to false negative or false positive findings. Secondly, the status of comorbidities should also be considered. ${ }^{6}$ In addition, although brain cortical development in children with ADHD lagged behind that of healthy children, children with ADHD might reach the peak cortical thickness several years later. ${ }^{26}$ So, the effects from development are needed to be investigated precisely in the future. In all, we need subsequent research with a larger sample size to evaluate the influence of comorbidities and development on cortical morphology.

\section{Cortical morphology abnormalities and WM}

In the current study, cortical volume in the left lateral STG was positively correlated with DSB scores in children with ADHD, suggesting that the left lateral STG might be associated with WM. Children with ADHD are likely to exhibit obvious WM deficits according to a number of studies. ${ }^{62,63}$ Fortunately, WM can be improved after working memory training with a commercially available and computerized working memory program (Cogmed) in children with ADHD. ${ }^{64}$ The STG is significantly associated with inattention among children with ADHD in one sMRI study. ${ }^{65}$ Meanwhile, WM deficits are strongly related to symptoms of inattention in children with ADHD. ${ }^{66}$ Lesions in lateral temporal lobes have 
been proved being able to disrupt WM processes. ${ }^{67} \mathrm{~A}$ recent study has claimed that human lateral temporal cortex is part of the neural system for verbal WM ${ }^{68}$ which is in line with our findings to some extent. To this day, there have been many fMRI studies confirming that WM in ADHD is associated with brain activation changes and abnormal functional connectivity, such as less activation in the left ventrolateral prefrontal cortex (VLPFC), cerebellar, occipital regions and lower connectivity in the ventrolateral prefrontal cortex, the ACC, the superior parietal lobule, and the cerebellum, ${ }^{63}$ less activity in right frontal, temporal, and subcortical regions and left occipital and cerebellar regions in male $\mathrm{ADHD}$ subjects during performance of a verbal working memory task, ${ }^{69}$ and Default Mode Network (DMN) functional abnormalities. ${ }^{70-72}$ Much research attention has been paid to the association between the prefrontal cortex and WM in $\mathrm{ADHD},{ }^{73-75}$ whereas this study should add to the evidence that ADHD deficits in WM are linked to several specific brain regions including left STG. ${ }^{76}$ However, future research with more detailed indicators of WM might help better understand the correlations involved. Furthermore, the correlation between cortical volume in the left lateral STG and DSB scores was only indicated in children with ADHD, but not in healthy controls. This might be related to several factors such as stronger variance in children with ADHD and clinical characteristics that are specific to the patients group.

In conclusion, this study confirms previous findings of cortical morphology abnormalities in children with ADHD, demonstrating that children with ADHD have regional cortical morphology abnormalities, especially smaller cortical volumes in the left lateral STG and left ACC. Moreover, volumes in the left lateral STG is associated with verbal WM. This study suggests that reduced cortical volumes are associated with $\mathrm{ADHD}$, and may be essential for understanding the pathophysiology of the disorder.

\section{Limitations}

Several limitations must be considered for the present study. First, because of the relatively small sample size of this study, it was difficult to analyze the effects of ADHD subtypes on the cortical morphology. Therefore, a larger sample size would be necessary to strengthen our results. Previous work has found brain structural differences between ADHD-C group and ADHD-I group, informing that different ADHD subtypes may be different phenotypes. ${ }^{56}$ In our study, although no main diagnostic effect was found for cortical thickness, the preliminary analyses for subtypes indicated that ADHD-I group showed significantly increased cortical thickness in the left superior temporal sulcus, when compared with ADHD-C group (data not shown). The expanded sample size in the future might also help us to illustrate the potential effects of sub- types on cortical morphology abnormalities in children with ADHD. Second, a gender-by-diagnosis interaction effect might exist on the volume of brain abnormalities, ${ }^{41}$ such as ACC. ${ }^{77}$ Although sex has been matched for two groups in this present study, a larger sample size may still be needed for betweengender comparisons to further illustrate the gender effects on cortical morphology. Third, we could not exclude the potential confounding contribution of LD and DBD to the results of the present study. Further investigation will be desired to evaluate the effects of comorbidities on cortical morphology.

\section{Supplementary Materials}

The online-only Data Supplement is available with this article at https://doi.org/10.30773/pi.2020.0333.

\section{Acknowledgments}

This work was supported by the National Science Foundation of China (81571340), the National Key Basic Research Program of China (973 program 2015CB856405), Beijing Municipal Science \&Technology Commission (No.Z161100000516032), the National Natural Science Foundation of China (81641163, 81761148026, 81873802), Beijing Natural Science Foundation (7172245), and the Capital Foundation of Medical Developments (CFMD:2016-2-4113). We thank all the participants for their kind contributions to our research. Thank Nan Li, Feng-Mei Fan, and Zhi-Jiang Wang for their help in statistical analyses.

\section{Conflicts of Interest}

The authors have no potential conflicts of interest to disclose.

\section{Author Contributions}

Conceptualization: Qiu-Jin Qian, Lu Liu. Data curation: Fei-Fei Si. Formal analysis: Fei-Fei Si, Lu Liu. Funding acquisition: Qiu-Jin Qian, Lu Liu. Methodology: Fei-Fei Si, Lu Liu, Hai-Mei Li, Li Sun, Qing-Jiu Cao. Project administration: Hai-Mei Li, Li Sun, Qing-Jiu Cao. Resources: Yu-Feng Wang, Li Sun, Qing-Jiu Cao. Software: Fei-Fei Si. Supervision: Qiu-Jin Qian, Lu Liu, Hanna Lu. Validation: Fei-Fei Si, Lu Liu. Writing_original draft: Fei-Fei Si. Writing—review \& editing: Qiu-Jin Qian, Lu Liu, Hanna Lu.

\section{ORCID iDs}

$\begin{array}{ll}\text { Fei-Fei Si } & \text { https://orcid.org/0000-0003-0512-5833 } \\ \text { Lu Liu } & \text { https://orcid.org/0000-0003-0194-1454 } \\ \text { Hai-Mei Li } & \text { https://orcid.org/0000-0002-1440-1951 } \\ \text { Li Sun } & \text { https://orcid.org/0000-0002-2330-6622 } \\ \text { Qing-Jiu Cao } & \text { https://orcid.org/0000-0001-7889-8138 } \\ \text { Hanna Lu } & \text { https://orcid.org/0000-0002-9090-258X } \\ \text { Yu-Feng Wang } & \text { https://orcid.org/0000-0002-1243-9198 } \\ \text { Qiu-Jin Qian } & \text { https://orcid.org/0000-0001-5060-3772 }\end{array}$

\section{REFERENCES}

1. Thomas R, Sanders S, Doust J, Beller E, Glasziou P. Prevalence of attention-deficit/hyperactivity disorder: a systematic review and meta-analysis. Pediatrics 2015;135:e994-e1001.

2. American Psychological Association. Diagnostic and Statistical Manual of Mental Disorders: DSM-5, 5th Edn. Arlington: American Psychiatric Publishing, Inc; 2013.

3. van Ewijk H, Heslenfeld DJ, Zwiers MP, Buitelaar JK, Oosterlaan J. Diffusion tensor imaging in attention deficit/hyperactivity disorder: a systematic review and meta-analysis. Neurosci Biobehav Rev 2012;36:10931106.

4. Gehricke JG, Kruggel F, Thampipop T, Alejo SD, Tatos E, Fallon J, et 
al. The brain anatomy of attention-deficit/hyperactivity disorder in young adults - a magnetic resonance imaging study. PLoS One 2017;12: e0175433.

5. Carmona S, Vilarroya O, Bielsa A, Trèmols V, Soliva JC, Rovira M, et al. Global and regional gray matter reductions in ADHD: a voxel-based morphometric study. Neurosci Lett 2005;389:88-93.

6. Noordermeer SDS, Luman M, Greven CU, Veroude K, Faraone SV, Hartman CA, et al. Structural brain abnormalities of attention-deficit/hyperactivity disorder with oppositional defiant disorder. Biol Psychiatry 2017;82: 642-650

7. Cubillo A, Halari R, Smith A, Taylor E, Rubia K. A review of fronto-striatal and fronto-cortical brain abnormalities in children and adults with Attention Deficit Hyperactivity Disorder (ADHD) and new evidence for dysfunction in adults with ADHD during motivation and attention. Cortex 2012;48:194-215.

8. Shaw P, Malek M, Watson B, Sharp W, Evans A, Greenstein D. Development of cortical surface area and gyrification in attention-deficit/hyperactivity disorder. Biol Psychiatry 2012;72:191-197.

9. Shaw P, Gilliam M, Liverpool M, Weddle C, Malek M, Sharp W, et al. Cortical development in typically developing children with symptoms of hyperactivity and impulsivity: support for a dimensional view of attention deficit hyperactivity disorder. Am J Psychiatry 2011;168:143151.

10. Batty MJ, Palaniyappan L, Scerif G, Groom MJ, Liddle EB, Liddle PF, et al. Morphological abnormalities in prefrontal surface area and thalamic volume in attention deficit/hyperactivity disorder. Psychiatry Res 2015; 233:225-232.

11. Wolosin SM, Richardson ME, Hennessey JG, Denckla MB, Mostofsky $\mathrm{SH}$. Abnormal cerebral cortex structure in children with ADHD. Hum Brain Mapp 2009;30:175-184.

12. Shaw P, Lerch J, Greenstein D, Sharp W, Clasen L, Evans A, et al. Longitudinal mapping of cortical thickness and clinical outcome in children and adolescents with attention-deficit/hyperactivity disorder. Arch Gen Psychiatry 2006;63:540-549.

13. Kumar U, Arya A, Agarwal V. Neural alterations in ADHD children as indicated by voxel-based cortical thickness and morphometry analysis. Brain Dev 2017;39:403-410.

14. Gau SS, Shang CY. Executive functions as endophenotypes in ADHD: evidence from the Cambridge Neuropsychological Test Battery (CANTAB). J Child Psychol Psychiatry 2010;51:838-849.

15. Haring L, Müürsepp A, Mõttus R, IlvesP, KochK, Uppin K, et al. Cortical thickness and surface area correlates with cognitive dysfunction among first-episode psychosis patients. Psychol Med 2016;46:21452155.

16. Hartberg CB, Sundet K, Rimol LM, Haukvik UK, Lange EH, Nesvåg R, et al. Brain cortical thickness and surface area correlates of neurocognitive performance in patients with schizophrenia, bipolar disorder, and healthy adults. J Int Neuropsychol Soc 2011;17:1080-1093.

17. Rodrigue AL, McDowell JE, Tandon N, Keshavan MS, Tamminga CA, Pearlson GD, et al. Multivariate relationships between cognition and brain anatomy across the psychosis spectrum. Biol Psychiatry Cogn Neurosci Neuroimaging 2018;3:992-1002.

18. Duan K, Chen J, Calhoun VD, Lin D, Jiang W, Franke B, et al. Neural correlates of cognitive function and symptoms in attention-deficit/hyperactivity disorder in adults. Neuroimage Clin 2018;19:374-383.

19. Cockcroft K. Working memory functioning in children with attentiondeficit/hyperactivity disorder (ADHD): a comparison between subtypes and normal controls. J Child Adolesc Ment Health 2011;23:107118.

20. Pastura G, Kubo TT, Regalla MA, Mesquita CM, Coutinho G, Gasparetto EL, et al. Working memory and left medial temporal cortical thickness. Arq Neuropsiquiatr 2016;74:785-790.

21. Narr KL, Woods RP, Lin J, Kim J, Phillips OR, Del'Homme M, et al. Widespread cortical thinning is a robust anatomical marker for attentiondeficit/hyperactivity disorder. J Am Acad Child Adolesc Psychiatry 2009;
48:1014-1022.

22. Saute R, Dabbs K, Jones JE, Jackson DC, Seidenberg M, Hermann BP. Brain morphology in children with epilepsy and ADHD. PLoS One 2014;9:e95269.

23. Dale AM, Fischl B, Sereno MI. Cortical surface-based analysis. I. Segmentation and surface reconstruction. Neuroimage 1999;9:179-194.

24. Fischl B, Sereno MI, Dale AM. Cortical surface-based analysis. II: Inflation, flattening, and a surface-based coordinate system. Neuroimage 1999;9:195-207.

25. Sasayama D, Hayashida A, Yamasue H, Harada Y, Kaneko T, Kasai K, et al. Neuroanatomical correlates of attention-deficit-hyperactivity disorder accounting for comorbid oppositional defiant disorder and conduct disorder. Psychiatry Clin Neurosci 2010,64:394-402.

26. Shaw P, Eckstrand K, Sharp W, Blumenthal J, Lerch JP, Greenstein D, et al. Attention-deficit/hyperactivity disorder is characterized by a delay in cortical maturation. Proc Natl Acad Sci U S A 2007;104:19649-19654.

27. Kaufman J, Birmaher B, Brent D, Rao U, Flynn C, Moreci P, et al. Schedule for Affective Disorders and Schizophrenia for School-Age ChildrenPresent and Lifetime Version (K-SADS-PL): initial reliability and validity data. J Am Acad Child Adolesc Psychiatry1997;36:980-988.

28. Gong YX, Cai TS. Chinese-Wechsler Intelligence Scale for Children. Hunan: Map Press; 1993.

29. Li XT. The distribution of left and right handedness in Chinese people. Acta Psychologica Sinica 1983;3:268-276.

30. Barkley RA, Fischer M, Smallish L, Fletcher K. The persistence of attention-deficit/hyperactivity disorder into young adulthood as a function of reporting source and definition of disorder. J Abnorm Psychol 2002; 111: 279-289.

31. Trampush JW, Jacobs MM, Hurd YL, Newcorn JH, Halperin JM. Moderator effects of working memory on the stability of ADHD symptoms by dopamine receptor gene polymorphisms during development. Dev Sci 2014;17:584-595.

32. Rosenthal EN, Riccio CA, Gsanger KM, Jarratt KP. Digit Span components as predictors of attention problems and executive functioning in children. Arch Clin Neuropsychol 2006;21:131-139.

33. Cassidy AR. Executive function and psychosocial adjustment in healthy children and adolescents: a latent variable modelling investigation. Child Neuropsychol 2016;22:292-317.

34. van Dongen-Boomsma M, Vollebregt MA, Buitelaar JK, Slaats-Willemse D. Working memory training in young children with ADHD: a randomized placebo-controlled trial. J Child Psychol Psychiatry 2014; 55:886-896.

35. Destrieux C, Fischl B, Dale A, Halgren E. Automatic parcellation of human cortical gyri and sulci using standard anatomical nomenclature. Neuroimage 2010;53:1-15.

36. Marschner IC. glm2: Fitting generalized linear models with convergence problems. R J 2011;3/2:12-15.

37. Vilgis V, Sun L, Chen J, Silk TJ, Vance A. Global and local grey matter reductions in boys with $\mathrm{ADHD}$ combined type and $\mathrm{ADHD}$ inattentive type. Psychiatry Res Neuroimaging 2016;254:119-126.

38. Villemonteix T, De Brito SA, Kavec M, Balériaux D, Metens T, Slama H, et al. Grey matter volumes in treatment naïve vs. chronically treated children with attention deficit/hyperactivity disorder: a combined approach. Eur Neuropsychopharmacol 2015;25:1118-1127.

39. Lim L, Marquand A, Cubillo AA, Smith AB, Chantiluke K, Simmons A, et al. Disorder-specific predictive classification of adolescents with attention deficit hyperactivity disorder (ADHD) relative to autism using structural magnetic resonance imaging. PLoS One 2013;8:e63660.

40. Chaim TM, Zhang T, Zanetti MV, da Silva MA, Louzã MR, Doshi J, et al. Multimodal magnetic resonance imaging study of treatment-naïve adults with attention-deficit/hyperactivity disorder. PLoS One 2014;9: e110199.

41. Seymour KE, Tang X, Crocetti D, Mostofsky SH, Miller MI, Rosch KS. Anomalous subcortical morphology in boys, but not girls, with ADHD compared to typically developing controls and correlates with emotion 
dysregulation. Psychiatry Res Neuroimaging 2017;261:20-28.

42. Hyun GJ, Jung TW, Park JH, Kang KD, Kim SM, Son YD, et al. Changes in gait balance and brain connectivity in response to equine-assisted activity and training in children with attention deficit hyperactivity disorder. J Altern Complement Med 2016;22:286-293.

43. Cao X, Cao Q, Long X, Sun L, Sui M, Zhu C, et al. Abnormal restingstate functional connectivity patterns of the putamen in medicationnaïve children with attention deficit hyperactivity disorder. Brain Res 2009;1303:195-206.

44. Rubia K, Smith AB, Brammer MJ, Taylor E. Temporal lobe dysfunction in medication-naïve boys with attention-deficit/hyperactivity disorder during attention allocation and its relation to response variability. Biol Psychiatry 2007;62:999-1006.

45. Shang CY, Yan CG, Lin HY, Tseng WY, Castellanos FX, Gau SS. Differential effects of methylphenidate and atomoxetine on intrinsic brain activity in children with attention deficit hyperactivity disorder. Psychol Med 2016;46:3173-3185.

46. Cerullo MA, Adler CM, Lamy M, Eliassen JC, Fleck DE, Strakowski $\mathrm{SM}$, et al. Differential brain activation during response inhibition in bipolar and attention-deficit hyperactivity disorders. Early Interv Psychiatry 2009;3:189-197.

47. Wolfers T, van Rooij D, Oosterlaan J, Heslenfeld D, Hartman CA, Hoekstra PJ, et al. Quantifying patterns of brain activity: distinguishing unaffected siblings from participants with ADHD and healthy individuals. Neuroimage Clin 2016;12:227-233.

48. Yang L, Wang YF, Qian QJ, Biederman J, Faraone SV. DSM-IV subtypes of ADHD in a Chinese outpatient sample. J Am Acad Child Adolesc Psychiatry 2004;43:248-250.

49. Mulas F, Capilla A, Fernández S, Etchepareborda MC, Campo P, Maestú F, et al. Shifting-related brain magnetic activity in attention-deficit/ hyperactivity disorder. Biol Psychiatry 2006;59:373-379.

50. Bonath B, Tegelbeckers J, Wilke M, Flechtner HH, Krauel K. Regional gray matter volume differences between adolescents with ADHD and typically developing controls: further evidence for anterior cingulate involvement. J Atten Disord 2018;22:627-638.

51. Frodl T, Skokauskas N. Meta-analysis of structural MRI studies in children and adults with attention deficit hyperactivity disorder indicates treatment effects. Acta Psychiatr Scand 2012;125:114-126.

52. Matsuo K, Nicoletti M, Nemoto K, Hatch JP, Peluso MA, Nery FG, et al. A voxel-based morphometry study of frontal gray matter correlates of impulsivity. Hum Brain Mapp 2009;30:1188-1195.

53. Mackie S, Shaw P, Lenroot R, Pierson R, Greenstein DK, Nugent TF 3rd, et al. Cerebellar development and clinical outcome in attention deficit hyperactivity disorder. Am J Psychiatry 2007;164:647-655.

54. Seidman LJ, Biederman J, Liang L, Valera EM, Monuteaux MC, Brown A, et al. Gray matter alterations in adults with attention-deficit/hyperactivity disorder identified by voxel based morphometry. Biol Psychiatry 2011;69:857-866.

55. Semrud-Clikeman M, Pliszka SR, Lancaster J, Liotti M. Volumetric MRI differences in treatment-naive vs chronically treated children with ADHD. Neurology 2006;67:1023-1027.

56. Semrud-Clikeman M, Fine JG, Bledsoe J, Zhu DC. Regional volumetric differences based on structural MRI in children with two subtypes of ADHD and controls. J Atten Disord 2017;21:1040-1049.

57. Tamm L, Menon V, Ringel J, Reiss AL. Event-related FMRI evidence of frontotemporal involvement in aberrant response inhibition and task switching in attention-deficit/hyperactivity disorder. J Am Acad Child Adolesc Psychiatry 2004;43:1430-1440.

58. Konrad K, Neufang S, Hanisch C, Fink GR, Herpertz-Dahlmann B. Dysfunctional attentional networks in children with attention deficit/ hyperactivity disorder: evidence from an event-related functional magnetic resonance imaging study. Biol Psychiatry 2006;59:643-651.

59. Bálint S, Bitter I, Czobor P. [Neurobiological correlates of cognitive flexibility in ADHD - A systematic review of the literature]. Psychiatr Hung
2015,30:363-371.

60. Janssen TWP, Heslenfeld DJ, van Mourik R, Maras A, Oosterlaan J. Alterations in the ventral attention network during the stop-signal task in children with ADHD: an event-related potential source imaging study. J Atten Disord 2018;22:639-650.

61. Yang XR, Carrey N, Bernier D, MacMaster FP. Cortical thickness in young treatment-naive children With ADHD. J Atten Disord 2015;19: 925-930.

62. Valera EM, Faraone SV, Biederman J, Poldrack RA, Seidman LJ. Functional neuroanatomy of working memory in adults with attention-deficit/hyperactivity disorder. Biol Psychiatry 2005;57:439-447.

63. Wolf RC, Plichta MM, Sambataro F, Fallgatter AJ, Jacob C, Lesch KP, et al. Regional brain activation changes and abnormal functional connectivity of the ventrolateral prefrontal cortex during working memory processing in adults with attention-deficit/hyperactivity disorder. Hum Brain Mapp 2009;30:2252-2266.

64. Kim HS, Lee EK, Hong IH, An JS, Yoo HK. Augmentative effects of working memory training on clinical symptoms and neuropsychology in medicated children and adolescents with attention-deficit hyperactivity disorder. Korean Acad Child Adolesc Psychiatry 2017;28:123-131.

65. McLaughlin KA, Sheridan MA, Winter W, Fox NA, Zeanah CH, Nelson CA. Widespread reductions in cortical thickness following severe early-life deprivation: a neurodevelopmental pathway to attention-deficit/hyperactivity disorder. Biol Psychiatry 2014;76:629-638.

66. Martinussen R, Tannock R. Working memory impairments in children with attention-deficit hyperactivity disorder with and without comorbid language learning disorders. J Clin Exp Neuropsychol 2006;28: 1073-1094.

67. Shallice T, Warrington EK. Independent functioning of verbal memory stores: a neuropsychological study. Q J Exp Psychol 1970;22:261-273.

68. Zamora L, Corina D, Ojemann G. Human temporal cortical single neuron activity during working memory maintenance. Neuropsychologia 2016;86:1-12.

69. Valera EM, Brown A, Biederman J, Faraone SV, Makris N, Monuteaux $\mathrm{MC}$, et al. Sex differences in the functional neuroanatomy of working memory in adults with ADHD. Am J Psychiatry 2010;167:86-94.

70. Silk T, Vance A, Rinehart N, Egan G, O’Boyle M, Bradshaw JL, et al. Fronto-parietal activation in attention-deficit hyperactivity disorder, combined type: functional magnetic resonance imaging study. Br J Psychiatry 2005;187:282-283.

71. Fassbender C, Zhang H, Buzy WM, Cortes CR, Mizuiri D, Beckett L, et al. A lack of default network suppression is linked to increased distractibility in ADHD. Brain Res2009;1273:114-128.

72. Biskup CS, Helmbold K, Baurmann D, Klasen M, Gaber TJ, BubenzerBusch S, et al. Resting state default mode network connectivity in children and adolescents with ADHD after acute tryptophan depletion. Acta Psychiatr Scand 2016;134:161-171.

73. Esposito F, Bertolino A, Scarabino T, Latorre V, Blasi G, Popolizio T, et al. Independent component model of the default-mode brain function: Assessing the impact of active thinking. Brain Res Bull 2006;70:263-269.

74. Sheridan MA, Hinshaw S, D'Esposito M. Efficiency of the prefrontal cortex during working memory in attention-deficit/hyperactivity disorder. J Am Acad Child Adolesc Psychiatry 2007;46:1357-1366.

75. Sheridan MA, Hinshaw S, D'Esposito M. Stimulant medication and prefrontal functional connectivity during working memory in ADHD: a preliminary report. J Atten Disord 2010;14:69-78.

76. Stevens MC, Pearlson GD, Kiehl KA. An FMRI auditory oddball study of combined-subtype attention deficit hyperactivity disorder. Am J Psychiatry 2007;164:1737-1749.

77. Villemonteix T, De Brito SA, Slama H, Kavec M, Balériaux D, Metens $\mathrm{T}$, et al. Grey matter volume differences associated with gender in children with attention-deficit/hyperactivity disorder: a voxel-based morphometry study. Dev Cogn Neurosci 2015;14:32-37. 
Supplementary Table 1. Estimated regression parameters, standard errors, $t$ values, and $p$-values for the group differences in cortical surface

\begin{tabular}{|c|c|c|c|c|}
\hline & Estimate & Std.error & t value & p-value \\
\hline \multicolumn{5}{|l|}{ Right hemisphere } \\
\hline Anterior cingulate cortex & 6.500 & 2.932 & 2.217 & 0.031 \\
\hline Supramarginal gyrus & 5.108 & 1.874 & 2.725 & 0.009 \\
\hline Pericallosal sulcus & 8.324 & 3.272 & 2.544 & 0.014 \\
\hline \multicolumn{5}{|l|}{ Left hemisphere } \\
\hline Anterior cingulate cortex & 8.254 & 2.545 & 3.244 & 0.002 \\
\hline Middle-anterior part of anterior cingulate cortex and sulcus & 8.971 & 3.375 & 2.658 & 0.010 \\
\hline Lateral aspect of the superior temporal gyrus & 1.374 & 4.420 & 3.110 & 0.003 \\
\hline Inferior segment of the circular sulcus of the insula & 1.719 & 5.920 & 2.904 & 0.005 \\
\hline Parieto-occipital sulcus & 5.936 & 2.711 & 2.190 & 0.003 \\
\hline
\end{tabular}

age, sex, full-scale intelligence quotient (FSIQ) and total intracranial volume (ICV) as covariates. All p-values implied uncorrected p-values 
Supplementary Table 2. Estimated regression parameters, standard errors, $\mathrm{t}$ values, and $\mathrm{p}$-values for the group differences in thickness

\begin{tabular}{|c|c|c|c|c|}
\hline & Estimate & Std.error & $\mathrm{t}$ value & p-value \\
\hline \multicolumn{5}{|l|}{ Right hemisphere } \\
\hline Inferior temporal gyrus & 1.074 & 3.315 & 3.241 & 0.002 \\
\hline Middle temporal gyrus & 6.970 & 2.896 & 2.406 & 0.020 \\
\hline Inferior frontal sulcus & 7.687 & 3.147 & 2.443 & 0.018 \\
\hline Anterior occipital sulcus and preoccipital notch & 9.036 & 3.253 & 2.778 & 0.007 \\
\hline Inferior temporal sulcus & 8.269 & 2.615 & 3.162 & 0.003 \\
\hline \multicolumn{5}{|l|}{ Left hemisphere } \\
\hline Short insular gyri & 7.648 & 3.177 & 2.407 & 0.020 \\
\hline Lateral aspect of the superior temporal gyrus & 7.167 & 2.875 & 2.493 & 0.016 \\
\hline
\end{tabular}

age, sex, full-scale intelligence quotient (FSIQ) and total intracranial volume (ICV) as covariates. All p-values implied uncorrected p-values 
Supplementary Table 3. Estimated regression parameters, standard errors, t values, and $p$-values for the group differences in cortical curvature

\begin{tabular}{lcccc}
\hline & Estimate & Std.error & t value & $\mathrm{p}^{-v a l u e^{\mathrm{a}}}$ \\
\hline Right orbital part of the inferior frontal gyrus & 5.934 & 2.249 & 2.639 & 0.011 \\
\hline
\end{tabular}

age, sex, full-scale intelligence quotient (FSIQ) and total intracranial volume (ICV) as covariates. All p-values implied uncorrected p-values 
Supplementary Table 4. The group differences in cortical volume among ADHD-I subtypes, ADHD-C subtypes and healthy controls

\begin{tabular}{lccccc}
\hline & $\mathrm{F}$ & $\mathrm{P}^{\mathrm{a}}$ & $\begin{array}{c}\text { ADHD-I vs. HC } \\
\left(\mathrm{p}^{\mathrm{a}}\right)\end{array}$ & $\begin{array}{c}\text { ADHD-C vs. HC } \\
\left(\mathrm{p}^{\mathrm{a}}\right)\end{array}$ & $\begin{array}{c}\text { ADHD-I vs. ADHD-C } \\
\left(\mathrm{p}^{\mathrm{a}}\right)\end{array}$ \\
\hline $\begin{array}{l}\text { Left lateral aspect of the superior } \\
\text { temporal gyrus }\end{array}$ & 13.369 & $1.40 \times 10^{-5 *}$ & $4.13 \times 10^{-4 *}$ & $1.25 \times 10^{-4 *}$ & 1.000 \\
\hline
\end{tabular}

age, sex, full-scale intelligence quotient (FSIQ) and total intracranial volume (ICV) as covariates. * p survived Bonferroni corrections. ADHD-I: attention-deficit/hyperactivity disorder inattentive type, ADHD-C: attention-deficit/hyperactivity disorder combined type, HC: healthy controls 


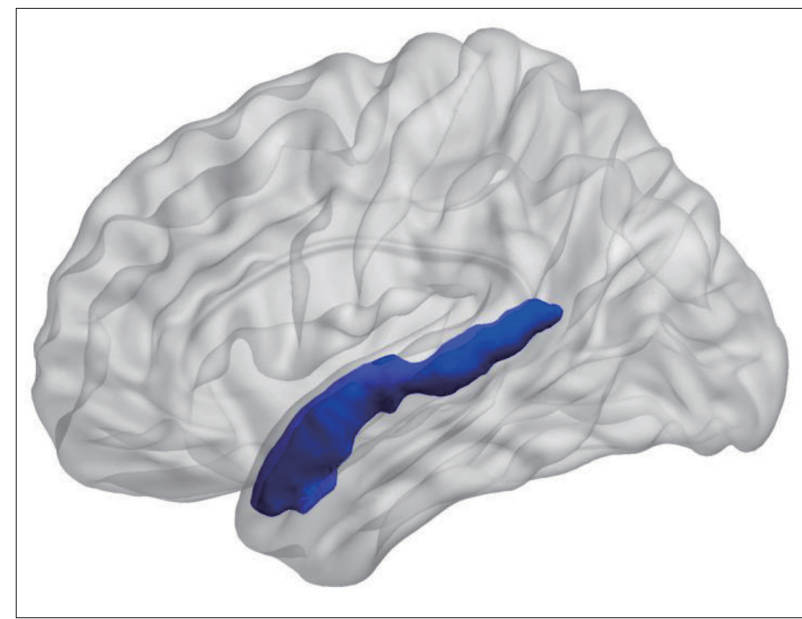

Supplementary Figure 1. The significant region of left lateral aspect of the superior temporal gyrus from $\mathrm{F}$ or post-hoc tests (both subgroups of ADHD vs. HC) is represented in blue color. The map is visualized by BrainNet Viewer version 1.7 (www.nitrc.org/ projects/bnv/). ADHD: attention-deficit/hyperactivity disorder, HC: healthy controls. 